POLIBETÁNICA

Núm. 39, pp. 133-149. México, 2015

\title{
RECUPERACIÓN E IDENTIFICACIÓN DE MACRORRESTOS ARQUEOBOTÁNICOS EN EL MUSEO NACIONAL DE LAS CULTURAS, CIUDAD DE MÉXICO
}

\section{RECOVERY AND IDENTIFICATION OF ARCHEOBOTANICAL MACROREMAINS OF THE NATIONAL MUSEUM OF CULTURES, MEXICO CITY}

\begin{abstract}
Iris G. Galván--Escobedo ${ }^{1}$, Aurora Montúfar-López ${ }^{2}$, Ebandro Uscanga-Mortera ${ }^{1}$, Edmundo García-Moya ${ }^{1}$, y Rodrigo Esparza-López ${ }^{3}$

${ }^{1}$ Posgrado en Botánica, Campus Montecillo, Colegio de Postgraduados. Carretera México-

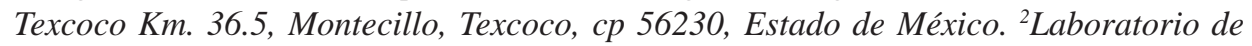
Paleobotánica, Subdirección de Laboratorios y Apoyo Académico, Instituto Nacional de Antropología e Historia. Moneda núm. 16, Col. Centro, cp 06060, México, DF. ${ }^{3}$ Centro de Estudios Arqueológicos, El Colegio de Michoacán. Martínez de Navarrete 505, Col.

Las Fuentes, Zamora, Michoacán, México.Correo electrónico: sirigales@gmail.com
\end{abstract}

\section{RESUMEN}

En el edificio que ocupa el Museo Nacional de las Culturas (MNC), del centro histórico de la ciudad de México, se llevaron a cabo excavaciones arqueológicas de las que se derivó esta investigación que tuvo como objetivo recuperar los restos arquebotánicos contenidos en los depósitos sedimentológicos de dichas excavaciones, con la finalidad de establecer las condiciones paleoecológicas de la región y el posible aprovechamiento de plantas. Se analizaron 50 muestras de sedimento que cronológicamente corresponden a finales de la época prehispánica y a la época colonial (siglos XVI a XIX). Los restos vegetales recuperados fueron: semillas y fragmentos de madera, que representaron a 36 géneros y una familia, de los cuales se pudo inferir el aprovechamiento de algunas plantas principalmente con fines alimenticio, medicinal, ceremonial y constructivo, así como algunas otras que aportan información de la vegetación de la región.

Palabras clave: macrorrestos botánicos, sedimentos, semillas, maderas, excavaciones arqueológicas.

ABSTRACT
Archeological excavations were carried
out in the building that houses the Natio-
nal Museum of Cultures (Museo Nacional
de las Culturas, MNC), which is located
in the historical center of Mexico City.
The present research derives from these
archeological works, whose objective was
the recovery of archaeobotanical remains
contained in the sediment deposits of such
excavations with the purpose of establish-
ing the paleoecological conditions of the
region and the possible use of plants. Fifty
sediment samples, which corresponded
chronologically to the end of the prehispa-


nic period and the beginning of the colonial era in Mexico (from the XVI to the XIX century), were analyzed. The vegetable remains recovered were seeds and wood fragments, which represented 36 genres and one family, and from which the use of some plants was inferred: food, medicine, ceremonial and building purposes. Other samples provided information about the vegetation in the region.

Key words: botanic macroremains, sediment, seeds, woods, archaeological excavations.

\section{INTRODUCCIÓN}

Los edificios coloniales del centro histórico de la Ciudad de México se erigieron sobre las ruinas de las construcciones prehispánicas que pertenecieron a la cultura Azteca, la cual habitó en la región de la cuenca de México a partir de 1325 y hasta el momento de su conquista por los españoles en 1521 .

Los aspectos de la organización social, política y económica de esta cultura y su posterior cambio como consecuencia del dominio de los españoles, se conocen gracias a los estudios arqueológicos realizados en la zona. De éstos, se han derivado investigaciones arqueobotánicas (Montúfar, 1991; 1996; 1998a; 1998b; 1998c; 1999; Montúfar y Valentín, 1998), que permiten identificar el aprovechamiento de las plantas en esta región durante la época prehispánica y la introducción de plantas por parte de los españoles durante la época colonial. Así mismo, los estudios arqueobotánicos, permiten hacer inferencias acerca de las modificaciones ocurridas en el ambiente en la zona durante los últimos 500 años.
Uno de los edificios que se construyeron en el centro histórico de la Ciudad de México durante el dominio de los españoles, es el que desde 1965 y hasta la fecha, alberga al Museo Nacional de las Culturas (MNC). El inmueble está construido sobre lo que, en la época prehispánica, fueron las Casas Nuevas del gobernante azteca Moctezuma (Almanza, 2009) y desde entonces se le asignaron diferentes usos, destacando el de Casa de Moneda Virreinal de 1734 a 1847 (Almanza, 2009).

Desde el 2002 hasta el 2010, un equipo de arqueólogos a cargo de la doctora Elsa Hernández Pons realizaron el proyecto arqueológico: "Coordinación Nacional de Monumentos Históricos-Museo Nacional de las Culturas (CNMH-MNC)", con el cual llevaron a cabo los trabajos necesarios para recuperar la mayor cantidad de información arqueológica e histórica relacionada con los diversos usos del predio del MNC en las épocas prehispánica, colonial y contemporánea, para plantear la restauración integral del edificio y su posterior adecuación museográfica (Hernández, 2004).

Durante el proceso de excavación, los arqueólogos tomaron muestras de sedimentos y, al mismo tiempo, se nos facilitó el acceso a las áreas de excavación para completar dicho muestreo. En total se recolectaron 50 muestras de sedimentos arqueológicos que corresponden a los periodos de ocupación de la época prehispánica y colonial del edificio del MNC. Los sedimentos se procesaron con el objetivo de recuperar e identificar los macrorrestos botánicos (semillas, frutos, flores, tallos, hojas, raíces, maderas, etc.) contenidos en ellos, para determinar la presencia de plantas que pudieron ser 
Galván Escobedo, I.G. et al.: Recuperación e identificación de macrorrestos arqueobotánicos, Museo Nal. de las Culturas, Cd. Méx.

utilizadas con fines alimenticio, medicinal, ceremonial o constructivo, y establecer las condiciones paleoecológicas en el sitio.

\section{Material y MÉtodos}

El MNC se localiza en el centro histórico de la ciudad de México, a un costado del Palacio Nacional, en la esquina de las calles de Moneda y Correo Mayor (fig. 1).

Dentro del museo, los arqueólogos definieron las diferentes zonas de excavación como "locales", cada uno identificado con la letra "A" y un número. Los resultados que se presentan, corresponden a las zonas exploradas arqueológicamente en los locales A-09, A-17, A-15 y A-23 (fig. 2).

Dentro de cada local, los arqueólogos abrieron de una a dos calas (área) de excavación en el subsuelo, en las cuales se registraron diferentes restos arqueológicos (cerámica, huesos, elementos constructivos, artefactos, etc.), lo que les permitió definir la temporalidad de cada una de las etapas de ocupación del espacio que alberga al actual edificio del museo. Dichos vestigios representan una ocupación del espacio del siglo XVI al XIX.

En el local A-09, se excavaron la cala 1 y cala 2a-b; en el local A-17, las calas 13 y $13 \mathrm{a}$, en el A-15, también conocidas como "Galería de los Monolitos", se excavó la cala "Pozo 2" y la cala "Elevador"; y finalmente, en el Local A-23, la cala "Los Comunes". En la cala 1, los arqueólogos definieron que los vestigios arqueológicos registrados, representan una ocupación del espacio en el siglo XVI, la cala 2a-b registró vestigios del siglo XVI al XVIII, la cala 13 , del siglo XVI, la 13 a del siglo
XVIII, el "Pozo 2" y "Elevador", de los siglos XVI al XIX y "Los Comunes", del XVIII y XIX. Como parte de los trabajos de las excavaciones arqueológicas se tomaron muestras de sedimentos para la recuperación de macrorrestos botánicos (semillas, frutos, hojas, tallos, etc.) en todas las calas mencionadas, ya que se aprovechó que en algunas excavaciones ya habían finalizado y aún se encontraban abiertas, o bien, estaban en proceso de excavación.

En los perfiles sedimentológicos de las calas 1, 2a-b, 13 y 13 a se tomaron 34 muestras (1 a 34) de sedimentos (fig. 3). Para evitar contaminación entre las muestras, éstas se obtuvieron iniciando en el fondo de la cala y continuando hacia arriba, cada $0.50 \mathrm{~m}$, a excepción de aquellas en las que la constitución del perfil (a veces conformado por materiales muy gruesos) no lo permitió de esta manera. Se utilizó una espátula cuadrada de metal para aflojar y desprender el sedimento del perfil, el cual se colocó en bolsas de polietileno etiquetadas con sus datos de número del local, el número de la cala, la orientación del perfil, el número de la muestra, su profundidad y la fecha. La cantidad de sedimento por muestra fue de $3 \mathrm{~kg}$ aproximadamente.

En las calas "Pozo 2", "Elevador" y "Los Comunes" no fue posible tomar muestras de los perfiles sedimentológicos, debido a que éstos estaban conformados por rellenos de materiales gruesos; por lo que las muestras que se procesaron fueron tomadas por los arqueólogos en estas áreas conforme se fue bajando el nivel de la excavación. En la cala "Pozo 2" se obtuvieron 10 muestras (de la 35 a 44), de la cala "Elevador", sólo una (muestra 45); y en la cala "Los Comunes" se tomaron cinco muestras (de 

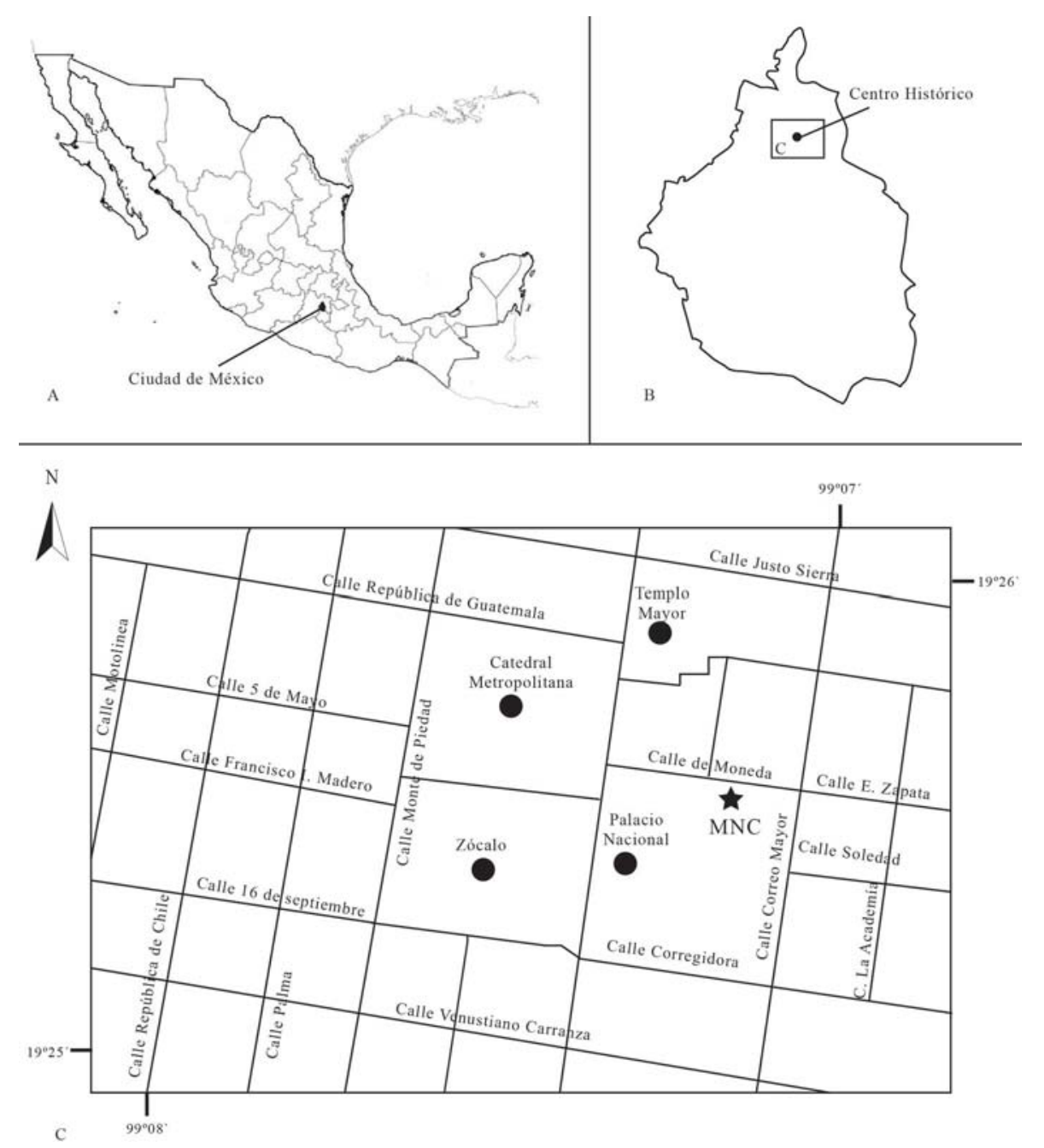

Fig. 1. Localización geográfica del Museo Nacional de las Culturas. A, Ubicación de la Ciudad de México. B, Localización del Museo Nacional de las Culturas en el centro histórico de la Ciudad de México. C, Detalle de la ubicación del Museo Nacional de las Culturas, Ciudad de México. 


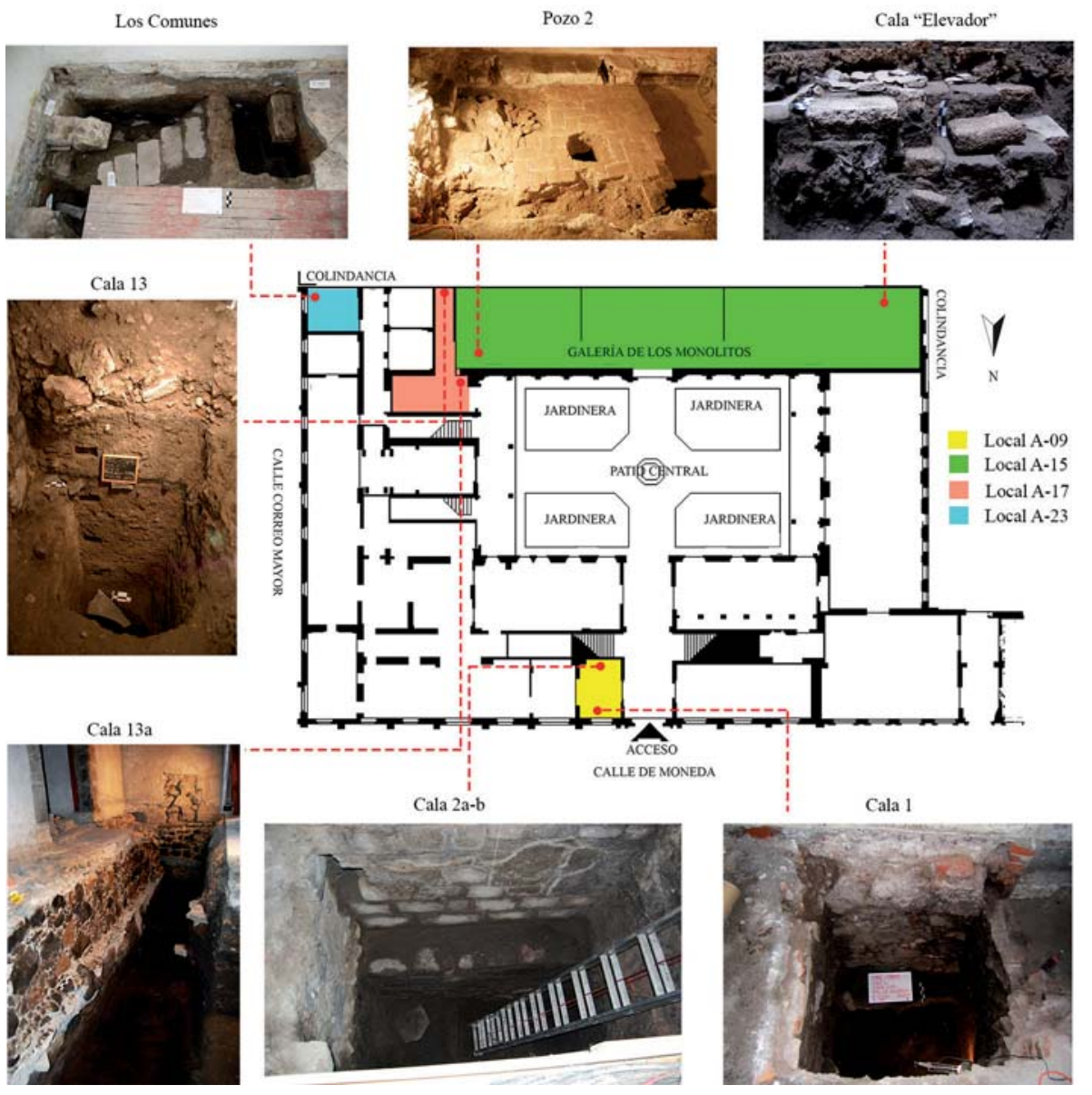

Fig. 2.Croquis de la planta baja del Museo Nacional de las Culturas y ubicación de las calas estudiadas (Croquis y fotografías cortesía del proyecto "CNMH-MNC" y de la doctora Elsa Hernández Pons). 

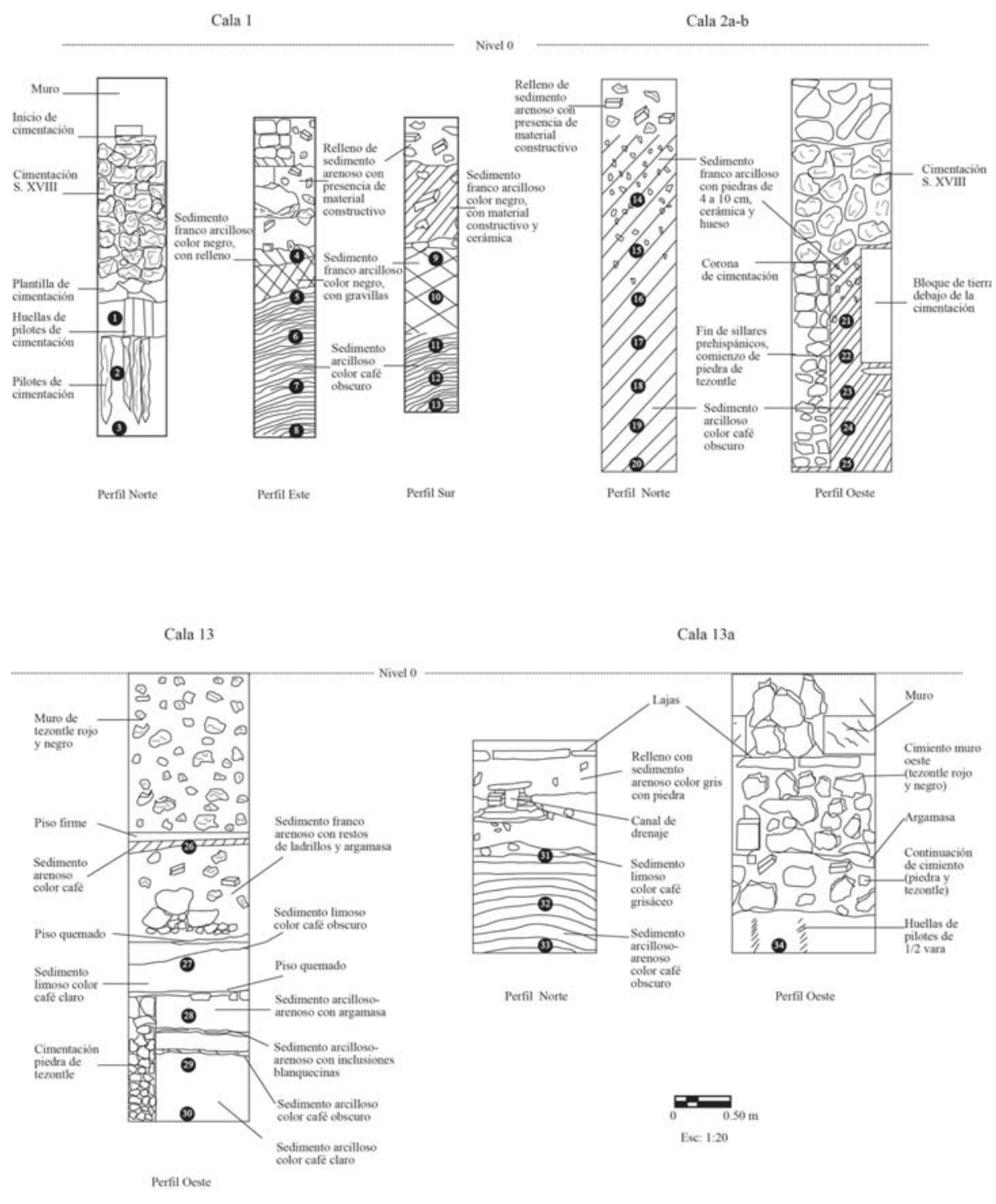

Fig. 3. Perfiles sedimentológicos de las excavaciones arqueológicas de las calas 1, 2a-b, 13 y 13a, en el Museo Nacional de las Culturas. Los círculos negros indican los puntos de muestreo para macrorrestos botánicos y número de las muestras (1-34). (Dibujos de perfiles cortesía del proyecto "CNMH-MNC"). 
la 46 a la 50). Entre las muestras que los arqueólogos recolectaron, se estudiaron seis fragmentos de maderas recuperados in situ en las calas 1 (muestras 55 y 56), 2a-b (muestra 53 y 54), 13a (muestra 51) y "Pozo 2" (muestra 52).

Las muestras se llevaron al Laboratorio de Paleobotánica de la Subdirección de Servicios Académicos del Instituto Nacional de Antropología e Historia (INAH). Para la recuperación de los macrorrestos botánicos, todas las muestras de sedimentos (excepto los fragmentos de maderas) fueron sometidas a la técnica de flotación en agua (Pearsall, 1989 modificada por Montúfar, 1996), la cual se basa en el principio de que los materiales con diferentes densidades se separan entre sí cuando se les coloca en un medio adecuado (en este caso agua simple) para su segregación, lo que hace posible la separación de las fracciones orgánicas e inorgánicas de la matriz del suelo y facilita la recuperación de material biológico que puede ser sujeto de análisis cuantitativos (Martínez, 1998).

La identificación de las semillas se hizo con ayuda de un microscopio estereoscópico, el cual permitió comparar las características físicas (forma, tamaño y ornamentación) con ejemplares ilustrados en la literatura especializada (Martín y Barkley, 1961; Davis, 1993; Espinosa y Sarukhán, 1997). Debido a que las características de las semillas suelen ser semejantes entre las especies, y a que gran parte de las semillas encontradas estuvieron fragmentadas, la identificación se realizó hasta el nivel de familia o género, y cuando fue posible, a especie.

De los fragmentos de maderas recuperados, se realizaron cortes histológicos manualmente y se hicieron preparaciones en fresco y sin tinción. Con un microscopio óptico y usando dos claves de identificación taxonómica, una propuesta por Camacho (1988), y la otra por García et al. (1996, 2003 y 2004), se identificaron las principales características anatómicas de las maderas para determinar el género al que pertenecieron.

\section{RESUltados}

En todas las muestras, excepto en la muestra 45 de la cala "Elevador" se recuperaron semillas y fragmentos de maderas (cuadro 1 y lámina 1). Ninguno de los macrorrestos estaba carbonizado. Los taxa de las semillas se identificaron: uno a nivel de familia, 30 a nivel de género, y tres al de especie. En las calas 1, 2a-b, 13, 13a y "Pozo 2" sobresale la presencia de semillas de arvenses, ruderales y viarias, tal es el caso de Oenothera sp. (hierba del golpe), Solanum sp. (duraznillo), Physalis sp. (tomatillo), Argemone sp. (chicalote), Potentilla sp. (periquito), Jaltomata sp. (jaltomate) y Asteraceae.

En las calas señaladas, también se registró la presencia de semillas de plantas de los géneros Scirpus sp. (tule), Ruppia sp., Potamogeton sp. (pasto de agua), Eleocharis sp. (junco) y Zannichellia sp. (espadaña). Por otro lado, se encontraron semillas de Sesuvium sp. (cenicienta o cenicilla) y Astragalus sp. (chinchines o sonadoras). La presencia de semillas de plantas alimenticias en dichas calas, se limitó a los taxa de Opuntia sp. (nopal), Crataegus mexicana DC. (tejocote), Cucurbita sp. (calabaza), Prunus persica (L.) Batsch (durazno) y Portulaca sp. (verdolaga). 
Cuadro 1. Macrorrestos botánicos (semillas y fragmentos de maderas) recuperados en las muestras de sedimento de las excavaciones arqueológicas del Museo Nacional de las Culturas.

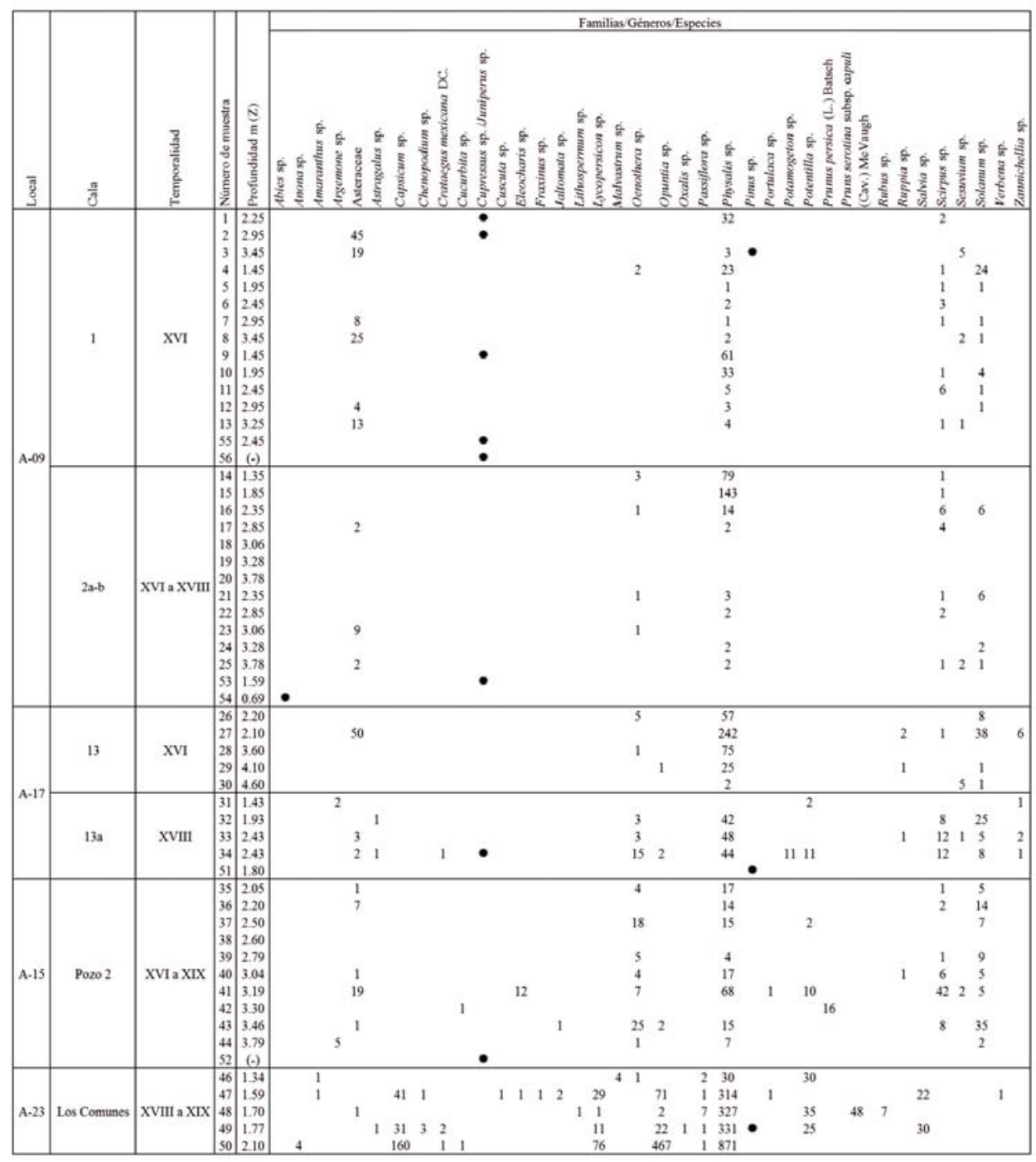

$(\bullet)$ = Presencia de fragmentos de madera $<1 \mathrm{~cm} ;(-)=$ Profundidad no indicada por los arqueólogos. 


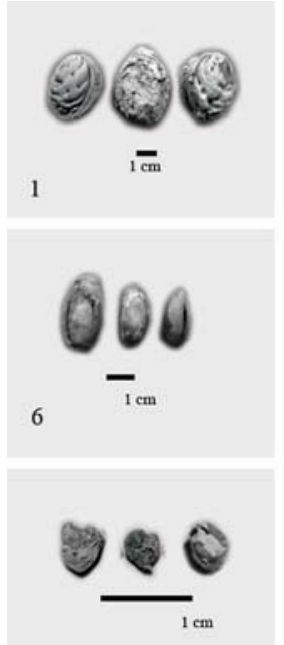

10

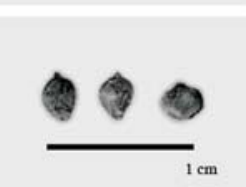

14

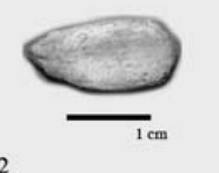

2

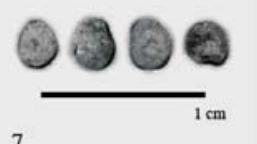

7

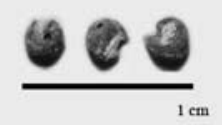

11

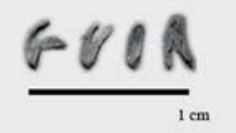

15

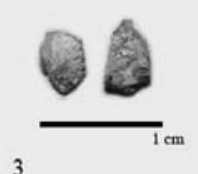

3
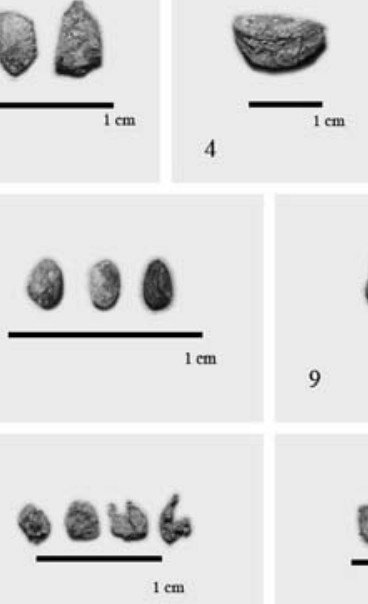

12

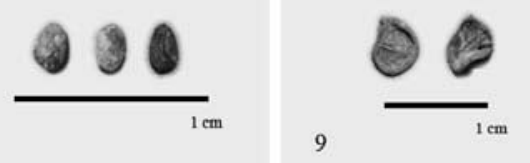

8
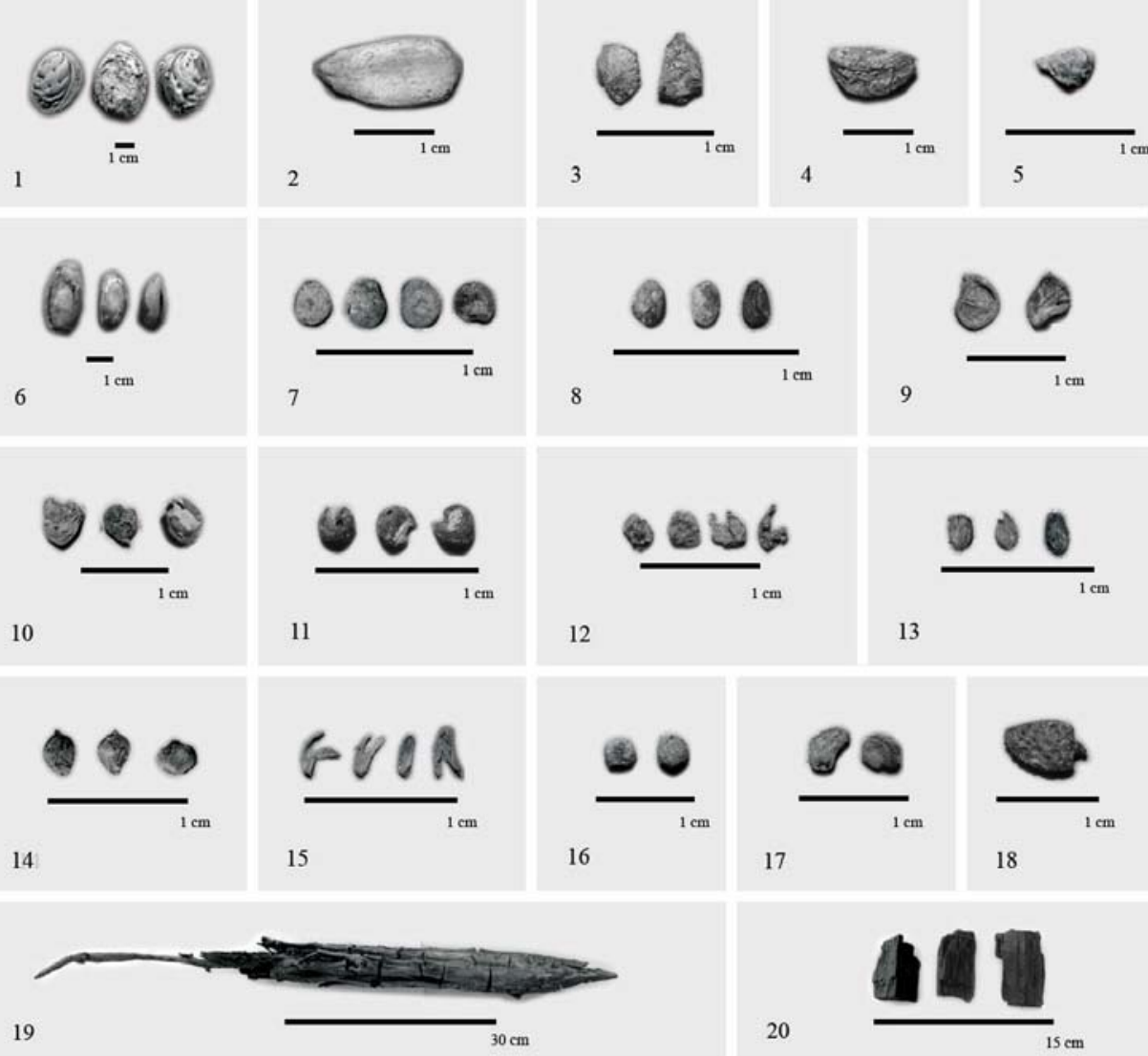

16

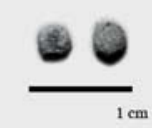

$1 \mathrm{~cm}$

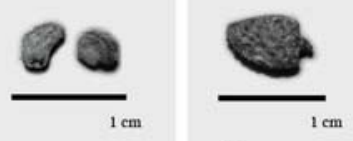

18

20
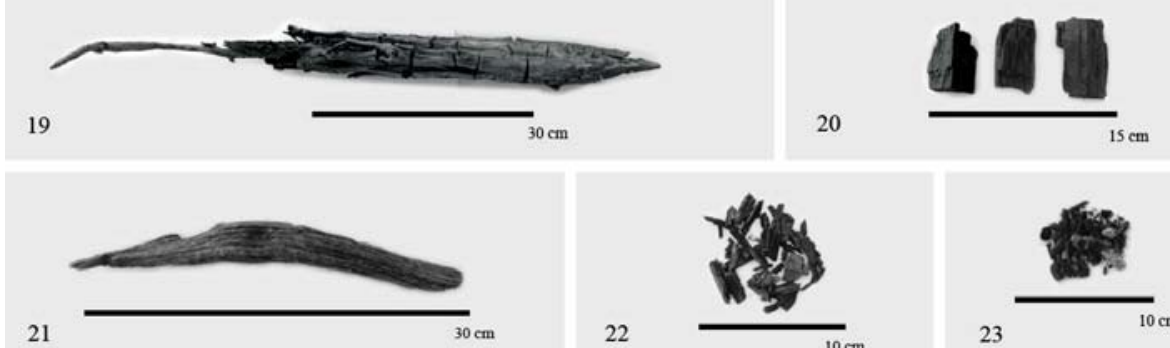

22
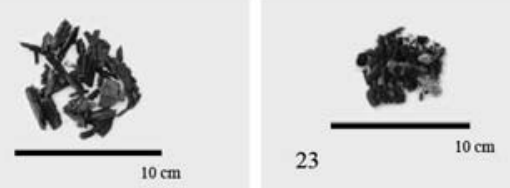

Lámina 1. Macrorrestos botánicos (semillas y fragmentos de maderas) recuperados en las excavaciones arqueológicas del Museo Nacional de las Culturas, ciudad de México. 1) Prunus persica (durazno); 2) Cucurbita sp. (calabaza); 3) Lycopersicon sp. (jitomate); 4) Crataegus mexicana (tejocote); 5) Lithospermum sp. (hierba de las perlitas); 6) Anona sp. (chirimoya); 7) Physalis sp. (tomate o tomatillo); 8) Salvia sp. (chía); 9) Capsicum sp. (chile); 10) Opuntia sp. (nopal); 11) Malvastrum sp. (chichichbe); 12) Solanum sp. (duraznillo); 13) Asteraceae; 14) Scirpus sp. (tule); 15) Eleocharis sp. (junco); 16) Argemone sp. (chicalote); 17) Potamogeton sp. (pasto de agua); 18) Passiflora sp. (granadilla); 19) Restos de un pilote de cimentación de Cupressaceae (cedro); 20) y 22) Fragmentos de Cupressaceae (cedro); 21) Fragmento de Pinus sp. (pino); 23) Fragmentos de Abies sp. (abeto). 
En la cala "Los Comunes" se recuperaron principalmente semillas de plantas alimenticias, medicinales o ceremoniales, y en menor proporción de arvenses, ruderales $\mathrm{y}$ viarias. Entre las plantas útiles que se encontraron están Anona sp. (chirimoya), Opuntia sp. (nopal), Cucurbita sp. (calabaza), Rubus sp. (zarzamora), Prunus serotina aff. subp. capuli (Cav.) McVaugh (capulín), Crataegus mexicana (tejocote), Capsicum sp. (chile), Lycopersicon sp. (jitomate), Passiflora sp. (granadilla), Physalis sp., Amaranthus sp. (quelite o amaranto), Chenopodium sp. (epazote), Portulaca sp. (verdolaga) y Salvia sp. (chía).

Entre las semillas de arvenses, ruderales y viarias de la cala "Los Comunes", destacan las mencionadas en las calas anteriores, además de las semillas de Lithospermum sp. (hierba de las perlitas), Cuscuta sp. (zacatlaxcale), Malvastrum sp. (Chichichbe), Oxalis sp. (agritos) y Verbena sp. Es importante señalar que en esta cala, la presencia de semillas pertenecientes a plantas de hábitos palustres fue poco significativa, pues sólo hubo una semilla del género Eleocharis sp. (junco).

Los fragmentos de maderas, se registraron en las calas 1, 2a-b, 12, 13a y "Pozo 2", y correspondieron a la familia Cupressaceae, género Cupressus sp. o Juniperus sp., y Pinaceae, géneros Pinus sp. y Abies sp.

\section{DISCUSIÓN}

Los taxa identificados se clasificaron en dos grupos. El primero, agrupa a las plantas que tienen uso alimenticio, medicinal, ceremonial y constructivo (cuadro 2). El segundo, agrupa aquellos taxa que denotan las características del hábitat como las arvenses, ruderales y viarias, plantas palustres, halófitas, así como las que habitan en bosques templados (cuadro 3), los cuales permitieron establecer algunas consideraciones ambientales.

\section{Plantas alimenticias, medicinales y ce- remoniales}

La presencia macrorrestos botánicos en "Los Comunes" fue particularmente significativa, ya que en dicha cala se recuperó la mayor cantidad de restos botánicos. En esta cala se registraron 17 de los 33 géneros encontrados en todo el muestreo en el MNC. Esto puede explicarse porque en esta zona se ubicaban los sanitarios de la antigua Casa de Moneda. Los lugares de desecho, como los sanitarios o basureros, suelen ser fuentes ricas en macrorrestos de plantas que se usaron como alimento humano.

La información del uso de plantas en la época prehispánica (Torres, 1989), así como los datos etnobotánicos actuales (Hernández-X., 1971; Martínez, 1969; García de Alba, 2012) permiten suponer que las semillas encontradas en "Los Comunes" son una evidencia del uso alimenticio, medicinal o ceremonial de las plantas por las personas que tuvieron contacto con el edificio del MNC, durante los siglos XVIII y XIX. Hay registros de que Amaranthus sp. (amaranto), Anona sp. (chirimoya), Capsicum sp. (chile), Chenopodium sp. (epazote), Crataegus mexicana (tejocote), Cucurbita sp. (calabaza), Lycopersicon sp. (jitomate), Opuntia sp. (nopal), Passiflora sp. (granadilla), Physalis sp. (tomatillo), Portulaca sp. (verdolaga), Prunus serotina aff. subsp. capuli (capulín) y Salvia sp. (chía) se consumían como alimento desde tiempos prehispánicos (Montúfar, 1998a; 1998c; 1999; 2013). 
Galván Escobedo, I.G. et al.: Recuperación e identificación de macrorrestos arqueobotánicos, Museo Nal. de las Culturas, Cd. Méx.

Cuadro 2. Agrupación de acuerdo al uso de los géneros de plantas registradas en las excavaciones arqueológicas del Museo Nacional de las Culturas.

\begin{tabular}{|c|c|c|c|}
\hline & Familia & Género & $\begin{array}{l}\begin{array}{l}\text { Nombre } \\
\text { común }\end{array} \\
\end{array}$ \\
\hline $\begin{array}{l}\text { Plantas de uso } \\
\text { alimenticio }\left({ }^{\mathrm{a}}\right) \text {, } \\
\text { medicinal }\left({ }^{\mathrm{m}}\right) \text { o } \\
\text { ceremonial }\left({ }^{\mathrm{c}}\right)\end{array}$ & $\begin{array}{l}\text { Anonaceae } \\
\text { Amaranthaceae } \\
\text { Cactaceae } \\
\text { Chenopodiaceae } \\
\text { Cucurbitaceae } \\
\text { Labiatae } \\
\text { Passifloraceae } \\
\text { Portulacaceae } \\
\text { Rosaceae }\end{array}$ & 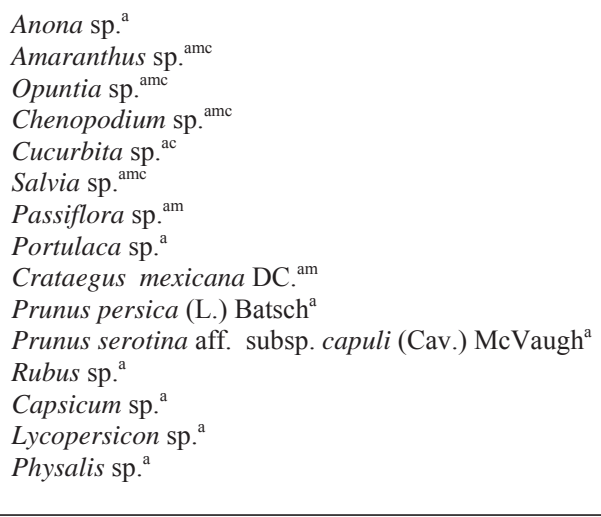 & $\begin{array}{l}\text { chirimoya } \\
\text { amaranto } \\
\text { nopal } \\
\text { quelite } \\
\text { calabaza } \\
\text { chía } \\
\text { granadilla } \\
\text { verdolaga } \\
\text { tejocote } \\
\text { durazno } \\
\text { capulín } \\
\text { zarzamora } \\
\text { chile } \\
\text { jitomate } \\
\text { tomate/ } \\
\text { tomatillo }\end{array}$ \\
\hline $\begin{array}{l}\text { Plantas usadas } \\
\text { en construcción }\end{array}$ & $\begin{array}{l}\text { Cupressaceae } \\
\text { Pinaceae }\end{array}$ & $\begin{array}{l}\text { Cupressus sp. /Juniperus sp. } \\
\text { Pinus } \mathrm{sp} . \\
\text { Abies } \mathrm{sp} .\end{array}$ & $\begin{array}{l}\text { cedro } \\
\text { pino } \\
\text { abeto }\end{array}$ \\
\hline
\end{tabular}

Algunas de estas plantas como Amaranthus sp. (amaranto), Chenopodium sp. (epazote), Crataegus mexicana (tejocote), Passiflora sp. (granadilla), Salvia sp. (chía) y Opuntia sp. (nopal) también son conocidas por su uso medicinal en la época prehispánica (Montúfar, 1998a; 1999), e incluso en el caso del amaranto, la chía y la calabaza, su uso se extendía al ceremonial, ya que se sabe que sus semillas se ofrendaban a los dioses relacionados con la fertilidad de la tierra y con el éxito de las cosechas (Montúfar, 2013).

Muchas de estas plantas eran cultivos habituales en los sistemas de producción agrícola prehispánicos, y muchas de ellas fueron domésticadas y consumidas en México desde hace aproximadamente 7000 años (por ejemplo Amaranthus sp., Capsicum sp., Cucurbita sp., Physalis sp. y Salvia sp.) (Montúfar, 2013). Al momento de la llegada de los españoles, los índigenas siguieron cultivando y utilizando las plantas que ellos conocían, pero también adoptaron las técnicas y cultivo de nuevas plantas introducidas por los españoles (Rojas-Rabiela, 2013). Por ejemplo, se ha documentado que con la llegada de los españoles, los campesinos indígenas aprendieron diversas técnicas como los injertos de manzano o durazno sobre patrones de tejocote, la cría de gusano de seda o el cultivo de plantas como el trigo y la caña (Rojas-Rabiela, 2013). 
Cuadro 3. Agrupación de acuerdo a su hábitat de los géneros de plantas encontradas en las excavaciones arqueológicas del Museo Nacional de las Culturas.

\begin{tabular}{|c|c|c|c|}
\hline & Familia & Género & Nombre común \\
\hline $\begin{array}{l}\text { Plantas arvenses, } \\
\text { ruderales y viarias }\end{array}$ & $\begin{array}{l}\text { Boraginaceae } \\
\text { Asteraceae } \\
\text { Convolvulaceae } \\
\text { Malvaceae } \\
\text { Onagraceae } \\
\text { Oxaladaceae } \\
\text { Papaveraceae } \\
\text { Rosaceae } \\
\text { Solanaceae } \\
\text { Verbenaceae }\end{array}$ & $\begin{array}{l}\text { Lithospermum sp. } \\
\text { No identificada } \\
\text { Cuscuta } \mathrm{sp} . \\
\text { Malvastrum } \mathrm{sp} . \\
\text { Oenothera } \mathrm{sp} . \\
\text { Oxalis } \mathrm{sp} . \\
\text { Argemone } \mathrm{sp} . \\
\text { Potentilla } \mathrm{sp} . \\
\text { Jaltomata } \mathrm{sp} . \\
\text { Solanum } \mathrm{sp} . \\
\text { Verbena } \mathrm{sp} .\end{array}$ & $\begin{array}{l}\text { hierba de las perlitas } \\
\text { zacatlaxcale } \\
\text { chichichbe } \\
\text { hierba del golpe } \\
\text { agritos } \\
\text { chicalote } \\
\text { periquito } \\
\text { jaltomate } \\
\text { duraznillo } \\
\text { verbena }\end{array}$ \\
\hline $\begin{array}{l}\text { Plantas de hábitos } \\
\text { palustres }\end{array}$ & $\begin{array}{l}\text { Cyperaceae } \\
\text { Potamogetonaceae } \\
\text { Zannichelliaceae }\end{array}$ & $\begin{array}{l}\text { Eleocharis } \mathrm{sp} . \\
\text { Scirpus } \mathrm{sp} . \\
\text { Potamogeton } \mathrm{sp} . \\
\text { Ruppia } \mathrm{sp} . \\
\text { Zannichellia } \mathrm{sp} .\end{array}$ & $\begin{array}{l}\text { junco } \\
\text { tule } \\
\text { pasto de agua } \\
\text { espadaña }\end{array}$ \\
\hline Plantas halófitas & Aizoaceae & Sesuvium sp. & cenicienta / cenicilla \\
\hline $\begin{array}{l}\text { Plantas que habitan } \\
\text { en climas templados }\end{array}$ & $\begin{array}{l}\text { Leguminosae } \\
\text { Cupressaceae } \\
\text { Pinaceae } \\
\text { Oleaceae }\end{array}$ & $\begin{array}{l}\text { Astragalus } \mathrm{sp} . \\
\text { Cupressus } \mathrm{sp} . \text { /Juniperus } \mathrm{sp} . \\
\text { Pinus } \mathrm{sp} . \\
\text { Abies } \mathrm{sp} . \\
\text { Fraxinus } \mathrm{sp} .\end{array}$ & $\begin{array}{l}\text { chinchines / sonadoras } \\
\text { cedro/ciprés } \\
\text { pino } \\
\text { abeto } \\
\text { fresno }\end{array}$ \\
\hline
\end{tabular}

El uso de plantas que se utilizaban en la época prehispánica y que se mantuvo durante la época colonial, se puede observar en las encontradas en "Los Comunes"; así mismo, es posible apreciar el uso de plantas introducidas por los españoles, como lo es el durazno (Prunus persica). Registros similares a los del MNC se han observado en los restos arqueobotánicos recuperados de las muestras de sedimentos provenientes de un basurero colonial en el Templo Ma- yor, del edificio Real Seminario de Minas, del predio 33 de la calle Justo Sierra (siglo XVII) y Palacio Nacional (siglo XVIII y principios del XIX); éste último localizado a un costado del MNC (Montúfar, 1998a; 1998b; Montúfar y Valentín, 1998).

Destaca que en los restos recuperados en el MNC no hubo presencia de semillas de Zea mays L. (maíz) o Phaseolus spp. (fríjoles), los cuales eran elementos importantes en 
la dieta de las personas durante la época prehispánica (y lo siguen siendo actualmente). Esto puede deberse al hecho de que dichas semillas tienen un bajo grado de preservación como consecuencia de que los tejidos que las constituyen resultan ser apetitosos para muchos insectos que se alimentan de ellas.

\section{Plantas útiles en la construcción}

Otro conjunto de plantas que destacó, principalmente en muestras procedentes de la cala 1 y la cala 13a, fue las empleadas en la construcción. Esta afirmación se desprende del hecho de que la mayoría de las muestras de madera se obtuvieron de sedimentos asociados a pilotes de madera utilizados como cimentación de los muros del MNC (cuadros 1 y 2).

Entre los géneros de madera que se identificaron está Cupressus sp. o Juniperus sp., a los cuales se les conoce comúnmente como "cedro blanco" o "ciprés". Actualmente ambos tienen importancia para la producción de madera y como ornamentales (Rzedowski y Rzedowski, 2005). Se trata de árboles de lugares templados, que crecen en alturas de 2000 a 3200 m.s.n.m., en sitios abrigados y húmedos, frecuentemente en las barrancas o en las orillas de los ríos. $\mathrm{Su}$ madera es de buena calidad y durable (Martínez, 1963). Estas características, además de que actualmente este género aún se encuentra distribuido en algunas regiones de la Cuenca de México, sugieren que fue un recurso disponible en la zona desde épocas remotas y que fue aprovechado en la construcción de los edificios coloniales, como lo evidencia su uso como pilotes de cimentación en la cala 1 .
Aunque no se tienen en este estudio registro de pilotes de Pinus sp. (pino), los fragmentos de madera identificados pertenecientes a este género, sugieren que probablemente también se utilizó en la elaboración de pilotes de construcción. Su uso para este fin, se registró en otras construcciones desde la época prehispánica y colonial, por ejemplo en el Templo Mayor (Montúfar, 1998a; 1998c) y otros sitios del centro histórico asociados a este centro ceremonial (Montúfar, 1991; 1999; Montúfar y Valentín, 1998).

Por último, si bien solamente se identificó una muestra de madera muy pequeña y a poca profundidad $(0.69 \mathrm{~m})$ en la cala $2 \mathrm{a}-\mathrm{b}$, como perteneciente al género Abies sp. (abeto), es posible que también se haya utilizado en la construcción, al igual que en otros sitios arqueológicos del centro histórico (Montufar, 1998a; 1998b).

\section{Consideraciones ambientales}

En las calas 1, 2a-b, 13, 13a y "Pozo 2" se encontraron principlamente restos de arvenses, ruderales y viarias, probablemente como consecuencia de la perturbación del ambiente lacustre que fue transformado en agrícola por la demanda de alimentos por parte de los pueblos indígenas, situación que se acentuó a partir de la llegada de los españoles y a la introducción del ganado (Rojas-Rabiela, 2013) en la cuenca de México.

Estos resultados coinciden con los de otros sitios del centro histórico de la ciudad de México, tal es el caso de los edificios 11 de la calle de Correo Mayor, el Real Seminario de Minas, un basurero colonial 
en el Templo Mayor y de la Catedral y el Sagrario Metropolitano (Montúfar, 1991; Montúfar, 1998b; Montúfar y Valentín, 1998; Montúfar, 1999). En estos estudios, los autores asocian la presencia de dichos géneros con la existencia de disturbios ecológicos en la zona ocasionados, entre otros factores, por las actividades del hombre como la agricultura y la urbanización.

Por otro lado, un gran número de semillas de Physalis sp. (tomate o tomatillo), se encontraron de 1 a $3 \mathrm{~m}$ de profundidad, situación semejante localizada en los sedimentos del subsuelo del edifico Real Seminario de Minas (Montúfar y Valentín, 1998) $\mathrm{y}$ en el edificio 11 de la calle de Correo Mayor de la ciudad de México (Montúfar, 1991). Los investigadores señalan que la alta proporción de estos restos vegetales coincide con periodos de alta precipitación $\mathrm{y}$ alta humedad ambiental que favorecieron el desarrollo de estas plantas y sus semillas fueron arrastradas a los lugares antes mencionados por las inundaciones durante la época colonial (siglos XVII al XVIII). En efecto, en las excavaciones arqueológicas del local A-15, a una profundidad no mayor de $2.46 \mathrm{~m}$, se localizaron abundantes caracoles de agua dulce en varias capas, lo cual fue interpretado por los arqueológos como evidencias de las inundaciones mencionadas (Almanza, 2009).

También destacó la presencia de plantas de hábitos palustres como Scirpus sp. (tule), Eleocharis sp. (junco), Potamogeton sp. (pasto de agua) y Ruppia sp., las cuales evidencian la influencia de los lagos que antiguamente conformaban la cuenca de México, y que han desaparecido junto con gran parte de las especies de plantas características de estos sitios. Esto también se refuerza con la presencia de Sesuvium sp. (cenicienta o cenicilla) que se desarrolla en suelos salinos.

La presencia en el registro arqueobotánico de maderas de Pinus sp. (pino), Cupressaceae (cedro), Abies sp. (abeto), semillas de Fraxinus sp. (fresno) y Astragalus sp. (chinchines o sonadoras) (ésta última, una planta que normalmente se le encuentra asociada a bosques de coníferas) sugieren que desde el siglo XVI hasta la fecha, la cuenca de México no ha registrado cambios importantes de clima (Montúfar y Valentín, 1998).

\section{Conclusiones}

En los depósitos sedimentológicos culturales del subsuelo del MNC se recuperaron los restos de la vegetación natural y la introducida por el hombre, que son una evidencia de la interacción hombre-planta-medio que se dio en el pasado. Se encontraron semillas de plantas de usos alimenticio, medicinal o ceremonial, principalmente en muestras provienentes de sedimentos que corresponden a los antiguos sanitarios ("Los Comunes") de la antigua Casa de Moneda (hoy MNC). Esto sugiere que, entre los productos que se utilizaban en la antigua Casa de Moneda en los siglos XVIII y XIX, se encontraban Anona sp. (chirimoya), Amaranthus sp. (amaranto), Opuntia sp. (nopal), Chenopodium sp. (epazote), Cucurbita sp. (calabaza), Salvia sp. (chía), Passiflora sp. (granadilla), Portulaca sp. (verdolaga), Crataegus mexicana (tejocote), Prunus persica (durazno), Prunus serotina aff. subsp. capuli (capulín), Rubus sp. (zarzamora), Capsicum sp. (chile), Lycopersicon sp. (jitomate) y Physalis sp. (tomate o tomatillo). Entre las plantas utilizadas en la construcción, se encontraron restos de maderas de Cupressus sp. o 
Juniperus sp. (cedro blanco o ciprés), Pinus sp. (pino), y Abies sp. (abeto), de los cuales se hizo evidente su uso como pilotes de cimentación.

Por otro lado, se registraron semillas que permitieron hacer inferencias acerca de las condiciones fisiográficas que predominaron en la cuenca de México en el pasado. Se encontraron restos de plantas de hábitos palustres como: Eleocharis sp. (junco), Scirpus sp. (tule), Potamogeton sp. (pasto de agua), Ruppia sp. y Zannichellia sp. (espadaña), atestiguando la abundancia de cuerpos de agua en la región. Los restos de plantas del género como Sesuvium sp. (cenicilla) manifiestan la presencia de los suelos salinos que circundaban a estas masas de agua.

Los restos de plantas arvenses, ruderales y viarias como Lithospermum sp. (hierba de las perlitas), Asteraceae, Cuscuta sp. (zacatlaxcale), Malvastrum sp. (chichichbe), Oenothera sp. (hierba del golpe), Oxalis sp. (agritos), Argemone sp. (chicalote), Potentilla sp. (periquito), Jaltomata sp. (jaltomate), Solanum sp. (duraznillo) y Verbena sp., son evidencia de prácticas agrícolas y, en general, de otras actividades humanas que conllevaron a la perturbación del ambiente. Por consiguiente, los paisajes agrícolas debieron predominar alrededor de los asentamientos humanos.

\section{AgRAdecimientos}

A la doctora Elsa Hernández Pons por su amplia disposición para permitir la toma de muestras de sedimento dentro del proyecto arqueológico "CNMH-MNC" y por las facilidades para tener acceso a los informes de excavación, ilustraciones y material bi- bliográfico utilizado en esta investigación. A la arqueóloga Colette Almanza por toda su ayuda y por fungir como enlace con el proyecto "CNMH-MNC". De igual manera a los arqueólogos Braulio Pérez, Georgina Ibarra y Angélica López. Al maestro en ciencias Alejandro Medina del INAH (ciudad de México) por su asesoría en el procesamiento e identificación de las muestras de madera. A la maestra en ciencias Petra Yáñez del Posgrado en Botánica del Colegio de Postgraduados por su valiosa colaboración.

\section{LITERATURA CITADA}

Almanza, C., 2009. “Arqueología de un proceso museográfico: La Galería de los Monolitos del Museo Nacional". Tesis de licenciatura de la Escuela Nacional de Antropología e Historia. 173 pp.

Camacho, D., 1988. La madera, estudio anatómico y catálogo de especies mexicanas. Colección Científica núm. 168. Instituto Nacional de Antropología e Historia. México. 364 pp.

Davis, L.W., 1993. Weed seeds of the Great Plains. A Handbook for identification. University Press of Kansas, United States of America. 145 pp.

Espinosa, F., y J. Sarukhán, 1997. Manual de Malezas del Valle de México. Universidad Nacional Autónoma de México y Fondo de Cultura Económica. México. 407 pp.

García, L.; A. Guindeo, y P. de Palacios. 1996. Maderas de coníferas. Anatomía de Géneros. Fundación Conde del Valle Salazar. Madrid, España. 349 pp. 
García, L.; A. Guindeo, C. Peraza, y P. de Palacios. 2003. La madera y su anatomía. Anomalías y defectos, estructura microscópica de coníferas y frondosas, identificación de maderas, descripción de especies y pared celular. Fundación Conde del Valle Salazar y Grupo Mundi-Prensa. Madrid, España. 327 pp.

García, L.; P. de Palacios, A. Guindeo y F. García, 2004. "Characterisation of the xylem of 352 conifers". Invest. Agrar. Sist. Recur. For., 13(3): 452-478.

García de Alba, J.E.; B. Ramírez, G. Robles, J. Zañudo, A.L. Salcedo, y J.E. García de Alba V., 2012. "Conocimiento y uso de las plantas medicinales en la zona metropolitana de Guadalajara". Desacatos, 39: 29-44.

Hernández-X., E., 1971. "Exploración etnobotánica y su metodología”. Xolocotzia, Revista de Geografía Agrícola, Universidad Chapingo, Tomo 1: 163-188.

Hernández, E., 2004. "Informe de sondeos arqueológicos en el Museo Nacional de las Culturas". $1^{a}$ parte. Informe Inédito. $8 \mathrm{pp}$.

Martin, C.A., y D.W. Barkley, 1961. Seed identification manual. University of California Press, California. 221 pp.

Martínez, M., 1963. Las pináceas mexicanas. $3^{\mathrm{a}}$ ed. Instituto de Biología. Universidad Nacional Autónoma de México. 400 pp.

1969. Las plantas medicinales de México. Ed. Botal 5a ed. México. 657 pp.
Martínez, D., 1998. Laboratorio de Paleobotánica y paleoambiente del IIA/ UNAM. Actualidades arqueológicas. No. 15-16. <http://morgan.iia.unam. $\mathrm{mx} / \mathrm{usr} /$ Actualidades/15/texto15/ paleoetnobotanica.html $>$ [21 de febrero de 2008].

Montúfar, A., 1991. "Estudio botánico de sedimentos arqueológicos, Correo Mayor 11, México, DF". Revista de Arqueología. Segunda época 21: 144-148.

1996. "Vegetación, Etnobotánica y Ambiente Prehispánico de Teotihuacán: Proyecto Interdisciplinario". Rev. Mex. Estud. Antropol., XLII: 63-69.

1998a. “Arqueobotánica del centro ceremonial de Tenochtitlán”. Arqueología Mexicana, VI(3):34-41.

,1998b. "Estudio botánico de un basurero colonial en el Templo Mayor, ciudad de México". Revista de Arqueología. Segunda época. 19: 173-177.

1998c. "Arqueobotánica de la ofrenda 000X del Templo Mayor". Resúmenes del III Congreso Mexicano de Etnobiología, "La etnobiología en el contexto de la globalización". Asociación etnobiológica Mexicana y el Instituto Tecnológico Agropecuario. Oaxaca, México. 64 pp.

1999. "Estudio arqueobotánico del subsuelo". Matos, E. (ed.). Excavaciones en la Catedral y el Sagrario metropolitano. Programa de arqueología urbana. Instituto Nacional de Antropología e Historia. México, DF.: 111-115. 
Galván Escobedo, I.G. et al.: Recuperación e identificación de macrorrestos arqueobotánicos, Museo Nal. de las Culturas, Cd. Méx.

Montúfar, A., y N. Valentín, 1998. "Estudio arqueobiológico de los sedimentos del subsuelo en el edificio Real Seminario de Minas, 1772, México, DF". Revista de Arqueología. Segunda época 20: 97-113.

2013. "Domesticación y cultivo de plantas alimenticias de México". Arqueología Mexicana, XIX(120): 42-47.

Pearsall, D.M., 1989. Paleoethnobotany. A handbook of procedures. Academic Press Inc., New York. 469 pp.

Rojas-Rabiela, T., 2013. "Agricultura colonial indígena". Arqueología mexicana, XIX(120): 62-67.
Rzedowski, G.C. de, J. Rzedowski et al., 2005. Flora fanerogámica del Valle de México. 2a. ed., Instituto de Ecología, A.C. y Comisión Nacional para el Conocimiento y Usos de la Biodiversidad, Pátzcuaro (Michoacán), 1406 pp.

Torres, B., 1989. "Las plantas útiles en el México antiguo según las fuentes del siglo XVI". Rojas-Rabiela, T. y W.T. Sanders, Historia de la agricultura, Época prehispánica Siglo XVI, Tomo I. Instituto Nacional de Antropología e Historia, México, DF.: 53-128.

Recibido: 8 junio 2013. Aceptado: 11 junio 2014. 